\section{Reply to M. Hertzberg et al}

Hertzberg et $\mathrm{al}^{1}$ commented on our report of etoposide, prednisone, vincristine, cyclophosphamide, doxorubicin, and rituximab (DA-EPOCH-R) in Burkitt lymphoma $(B L)^{2}$ The diagnosis of $B L$ is relatively straight forward because of its readily identifiable histology and immunophenotype and the MYC translocation, which is optional in the WHO diagnostic criteria. ${ }^{3}$ Among 65 centrally reviewed pathology reports from our study, four patient cases (6\%) did not have confirmation of a MYC translocation but had a classic histologic appearance, with Ki67 > 95\% and BCL2 negative. Thus, it is highly unlikely that our series included a large number of misdiagnosed cases, as suggested by the authors. In contrast, the authors present an inaccurate description of the published literature to support their concern. The first reference cited was described as a report using central pathology review, but it is actually a letter to the editor on a retrospective series of 145 patients with BL, Burkitt leukemia, or highgrade B-cell lymphomas that focuses on the poor outcomes of relapsed disease and does not address pathologic review. ${ }^{4}$ The second citation asserts that the rate of reproducibility of $\mathrm{BL}$ diagnosis is $53 \%$, but the study is mischaracterized. ${ }^{5}$ In fact, the retrospective study of 1,378 cases of lymphoma diagnosed three decades ago included only 10 patient cases of $\mathrm{BL}$, which were all confirmed on secondary review. The series also included 29 patient cases originally diagnosed as "high-grade B-cell, Burkittlike lymphoma," which had an agreement rate of $53 \%$ with secondary pathology review and which are no longer recognized diagnoses. In contrast, an accurate estimate according to contemporary diagnostic criteria comes from a study of 260 adult patients, which confirmed $\mathrm{BL}$ in $90 \%$ of cases on central pathology review. ${ }^{6}$ Finally, it is notable that the GMALL study, which the authors cite for comparison with our results, included Burkitt-like lymphoma and atypical Burkitt lymphoma, many pathological characteristics of which are not consistent with $\mathrm{BL}$, as well as Burkitt leukemia.?

The authors raise concerns related to the definition of high risk used in our study. There are no universally accepted definitions of disease risk in $\mathrm{BL}$, making cross-comparisons difficult. We used a definition based on stage, lactate dehydrogenase level, performance status, and mass size, similar to other studies. ${ }^{8}$ The percentages of patients with high risk according to the International Prognostic Index in our and the GMALL studies were similar: $45 \%$ and
$51 \%$, respectively. When comparing results of the DA-EPOCH-R and GMALL studies, the authors noted

5-year outcomes of $81.5 \%$ (event-free survival) and $90 \%$ (overall survival [OS]), respectively, for patients with an IPI of $0-2$, suggesting that DA-EPOCH-R was less effective. However, the GMALL study included pediatric patients where among patients age $16-25$ years versus $>25$ years, the OS was $90 \%$ versus 62\%-64\%, respectively, indicating a major influence of age on outcome. Importantly, the percent of patients $>55$ years old in the DA-EPOCH-R study was $42 \%$ compared with only $27 \%$ in the GMALL study-for which the progression-free survival was $60 \%$. It is notable that the authors make no mention of the equivalent efficacy of DA-EPOCH-R across age groups and ignore the notable adverse effect of age in the GMALL study. It is also troubling that the authors did not compare the outcomes of patients with high-risk IPI scores, for which DA-EPOCH-R showed an event-free survival rate of $88.2 \%$ and OS of $92.1 \% \mathrm{~W}$. Wilson, personal communication, August 2020) compared with an OS of $75 \%$ in the GMALL study. The suggestion that patients with $\mathrm{CNS} /$ bone marrow disease fared better in the GMALL study is also problematic, as the outcomes of $58.6 \%$ (event-free survival with DA-EPOCH-R) versus $67 \%$ (OS with GMALL) are within the confidence intervals and all patients with CNS disease in GMALL received radiotherapy. We point out these selective and inaccurate comparisons by the authors to emphasize the pitfalls of comparing greatly disparate trials and the importance of careful and thoughtful analyses.

We believe that a strong argument in favor of DA$\mathrm{EPOCH}-\mathrm{R}$ over dose-intense regimens is its riskbenefit in adult BL. Oddly, the authors imply that this may be untrue, because only " $82 \%$ completed the full protocol." ${ }^{1}$ In actuality, only five patients (5\%) with high-risk disease stopped DA-EPOCH-R early because of toxicity. One patient with low-risk disease abbreviated therapy because of toxicity. The notion that various regimens are equally tolerable lacks credibility. Indeed, the toxicity profile of any regimen is associated with age and performance status of the patient, which is why toxicity was separately reported for patients younger than 55 years in the GMALL study-but not in the DA-EPOCH-R study. ${ }^{9}$ Thus, we stand by our findings that DA-EPOCH-R compares favorably to more intensive regimens, but we recognize that improved therapeutic strategies are needed for adults with CSF involvement as well as careful consideration of treatment choices when treating highly curable diseases. 
However, we would like to emphasize that mischaracterizations and inappropriate comparisons of trials are of no service to patients or the medical community.

\section{Mark Roschewski, MD}

Lymphoid Malignancies Branch, National Cancer Institute, Bethesda, MD

\section{Kieron Dunleavy, MD}

George Washington University Cancer Center, Washington, $D C$

\section{Wyndham H. Wilson, MD, PhD}

Lymphoid Malignancies Branch, National Cancer Institute, Bethesda, MD

\section{AUTHORS' DISCLOSURES OF POTENTIAL CONFLICTS OF INTEREST AND DATA AVAILABILITY STATEMENT}

Disclosures provided by the authors and data availability statement (if applicable) are available with this article at DOI https://doi.org/10.1200/ JC0.20.02290.

\section{AUTHOR CONTRIBUTIONS}

Conception and design: All authors

Collection and assembly of data: Mark Roschewski

Data analysis and interpretation: Mark Roschewski

Manuscript writing: All authors

Final approval of manuscript: All authors

Accountable for all aspects of the work: All authors

\section{REFERENCES}

1. Hertzberg M, Joske DJL, Gandhi MK: DA-EPOCH-R in Burkitt lymphoma: Is it enough for high-risk disease? J Clin Oncol 38:3722-3723, 2020

2. Roschewski M, Dunleavy K, Abramson JS, et al: Multicenter study of riskadapted therapy with dose-adjusted EPOCH-R in adults with untreated Burkitt lymphoma. J Clin Oncol 38:2519-2529, 2020

3. Swerdlow S, Campo E, Harris N, et al: WHO Classification of Tumours of Haematopoietic and Lymphoid Tissues, Volume 2 (revised ed 4). Lyon, France, IARC, 2017.

4. Short NJ, Kantarjian HM, Ko H, et al: Outcomes of adults with relapsed or refractory Burkitt and high-grade B-cell leukemia/lymphoma. Am J Hematol 92:E114-E117, 2017 https://doi.org/10.1002/ajh.24720

5. The Non-Hodgkin's Lymphoma Classification Project: A clinical evaluation of the International Lymphoma Study Group classification of non-Hodgkin's lymphoma. Blood 89:3909-3918, 1997

6. Ribrag V, Koscielny S, Bosq J, et al: Rituximab and dose-dense chemotherapy for adults with Burkitt's lymphoma: A randomised, controlled, openlabel, phase 3 trial. Lancet 387:2402-2411, 2016

7. Hoelzer D, Walewski J, Döhner H, et al: Improved outcome of adult Burkitt lymphoma/leukemia with rituximab and chemotherapy: Report of a large prospective multicenter trial. Blood 124:3870-3879, 2014

8. Mead GM, Sydes MR, Walewski J, et al: An international evaluation of CODOX-M and CODOX-M alternating with IVAC in adult Burkitt's lymphoma: Results of United Kingdom Lymphoma Group LY06 study. Ann Oncol 13:1264-1274, 2002

9. Evens AM, Danilov AV, Jagadeesh D, et al: Burkitt lymphoma in the modern era: Real world outcomes and prognostication across 30 US cancer centers. Blood doi: 10.1182/blood.2020006926 [epub ahead of print on July 14, 2020]

DOI: https://doi.org/10.1200/JC0.20.02290; Published at ascopubs.org/ journal/jco on September 15, 2020. 
AUTHORS' DISCLOSURES OF POTENTIAL CONFLICTS OF INTEREST

\section{Reply to M. Hertzberg et al}

The following represents disclosure information provided by authors of this manuscript. All relationships are considered compensated unless otherwise noted. Relationships are self-held unless noted. I = Immediate Family Member, Inst = My Institution. Relationships may not relate to the subject matter of this manuscript. For more information about ASCO's conflict of interest policy, please refer to www.asco.org/rwc or ascopubs.org/jco/authors/author-center.

Open Payments is a public database containing information reported by companies about payments made to US-licensed physicians (Open Payments).

Kieron Dunleavy

Honoraria: AbbVie, Celgene, Karyopharm Therapeutics, Seattle Genetics,

AstraZeneca, Amgen, Kymera, Atara

Consulting or Advisory Role: Seattle Genetics, Karyopharm Therapeutics,

Celgene, AbbVie, AstraZeneca, Kymera, Atara, Amgen

No other potential conflicts of interest were reported. 